\title{
LOCALITY OF THE CONSERVATION LAWS FOR THE SOLITON EQUATIONS RELATED TO CAUDREY-BEALS-COIFMAN SYSTEM
}

\author{
ALEXANDAR YANOVSKI
}

Communicated by Metin Gürses

\begin{abstract}
We consider the hierarchies of Nonlinear Evolution Equations related to auxiliary problem of Caudrey-Beals-Coifman type. We give a proof that the conservation laws for these equations have local densities based on the theory of the generating operators related to the Caudrey-Beals-Coifman linear problem.
\end{abstract}

\section{Contents}

$\begin{array}{llr}1 & \text { Introduction } & 91\end{array}$

2 Fundamental Solutions to the CBC System $\quad 94$

3 Generating Operator Approach to the Soliton Equations Associated with the CBC System 95

3.1 Expansions Over the Adjoint Solutions for the CBC System . . . . . . . 95

4 The NLEEs Related to the CBC System 97

4.1 General Description . . . . . . . . . . . . . . . . . . . . . . 97

4.2 Conservation laws, Hamiltonian structures . . . . . . . . . . . . . . . 98

4.2.1. Properties of the Adjoint Solutions $h_{\nu, H} \ldots \ldots \ldots$. . . . . 98

4.2.2. Conservation Laws. The Generating Functions . . . . . . . . 100

5 Locality of the Hierarchy of NLEEs Related to the CBC System and Their Conservation Laws

References

106

\section{Introduction}

This article is about the theory of the so-called soliton equations (completely integrable equations). Their characteristic property is that they can be cast into the so called Lax form, or zero curvature form, that is, as compatibility condition (Lax 
representation) $[L, A]=0$ for two linear systems

$$
\begin{aligned}
& L \psi=\left(\mathrm{i} \partial_{x}-U\left(q, q_{x}, \ldots, \lambda\right)\right) \psi=0 \\
& A \psi=\left(\mathrm{i} \partial_{t}-V\left(q, q_{x}, \ldots, \lambda\right)\right) \psi=0 .
\end{aligned}
$$

Here $U, V$ are matrix functions, depending on the spectral parameter $\lambda$ and on a set of 'potential functions' $q(x, t) \equiv\left(q_{1}(x, t), q_{2}(x, t), \ldots, q_{N}(x, t)\right)$ and their spatial derivatives $q_{x}, q_{x x}, \ldots$ and $t$ is the time. The equation $[L, A]=0$ is equivalent to an equation (system) of the type $q_{t}=f\left(q, q_{x}, \ldots\right)$ which is the soliton equation itself, $[7,9,20]$. Usually the first of the equations in (1), that is $L \psi=0$, is fixed and called auxiliary linear problem. Changing the second one we obtain hierarchies of nonlinear evolution equations (NLEEs) related to the linear problem $L \psi=0$. Each hierarchy is usually named by some of the equations belonging to it.

Since the Lax representation is written as a commutator the theory of the NLEEs having Lax representation naturally uses classical theory of Lie algebras and in particular the semisimple Lie algebras theory. We shall suppose that the reader is familiar with it so we shall just introduce the notation we use, the one that is classical, see for example [13], we shall assume clear. For the semisimple Lie algebra $\mathfrak{g}$ over $\mathbb{C}$ we shall denote its Killing form by $\langle\rangle:,\langle X, Y\rangle=\operatorname{tr}\left(\operatorname{ad}_{X}, \operatorname{ad}_{Y}\right)$, $X, Y \in \mathfrak{g}$ where $\operatorname{ad}_{X}(Y) \equiv[X, Y]$.

We shall consider a fixed Cartan subalgebra $\mathfrak{h} \subset \mathfrak{g}$ and shall assume that the rank of $\mathfrak{g}$ is $r$, that is $\operatorname{dim} \mathfrak{h}=r$. The algebra is split into the following sum of subspaces mutually orthogonal with respect to the Killing form

$$
\mathfrak{g}=\mathfrak{h} \oplus \overline{\mathfrak{g}}, \quad \overline{\mathfrak{g}} \equiv \oplus_{\alpha \in \Delta} \mathbb{C} E_{\alpha} .
$$

The vectors $E_{\alpha}$ are the so-called root vectors, they are labeled by $\alpha \in \Delta$ where $\Delta$ (the set of roots) is a subset of $\mathfrak{h}^{*}-$ the dual of $\mathfrak{h}$. If we have some ordering on $\Delta$ it is possible to split $\Delta$ into the set of positive $\Delta_{+}$and negative $\Delta_{-}$roots $\Delta=$ $\Delta_{+} \cup \Delta_{-}$. Later we shall introduce orderings that are relevant for our tasks, now we only note that when some ordering is fixed one can introduce the set of simple roots $\pi=\left\{\alpha_{j}, 1 \leq j \leq r\right\}$, that is the positive roots that cannot be written as a sum of two positive roots. The vectors $\left\{E_{\alpha}, \alpha \in \Delta ; H_{i} \equiv\left[E_{\alpha_{i}}, E_{-\alpha_{i}}\right], \alpha_{i} \in \pi\right\}$ form a basis in $\mathfrak{g}$ called the Cartan-Weyl basis.

Let us return now to the Lax representations. Classical examples of auxiliary systems $L$ are the so called Zakharov-Shabat (ZS) system and its generalizations. The $\mathrm{ZS}$ system is of course the simplest one, since it is realized on $2 \times 2$ matrices. Its generalizations are quite natural from Lie algebra view point. We must take $L$ to be

$$
L \psi=\left(\mathrm{i} \partial_{x}+q(x)-\lambda J\right) \psi=0 .
$$


Here both $J$ and $q(x)$ belong to some fixed semisimple Lie algebra $\mathfrak{g}$ in some fixed faithful representation. The element $J$ is constant and regular so it defines a Cartan subalgeba $\mathfrak{h}=\operatorname{kerad}_{J}$. We shall consider it fixed. The 'potential' $q(x)$ is smooth $\overline{\mathfrak{g}}$-valued function. We obtain the ZS system for $\mathfrak{g}=\mathfrak{s l}(2, \mathbb{C})$ in the simplest representation and $J=\operatorname{diag}(1,-1)$.

The generalization of the Zakharov-Shabat system is evident from Lie algebra view point but it is not so easy to perform the generalization of the analytical results related to it. Those results are fundamental for the inverse scattering theory and it took several years to do it. From the beginning Zakharov and Shabat investigated the case $\mathfrak{g}=\mathfrak{s l}(n, \mathbb{C})$ in the simplest representation for real $J,[16,19,20]$ and later it was considered the case of arbitrary algebra in arbitrary finite dimensional faithful representation for real $J$, [8]. In case $J$ is real we shall call the system (3) the generalized Zakharov-Shabat (GZS) system.

Soon it became evident that the case when $J$ is complex is also quite interesting. One of the motivations to consider complex $J$ is that if one wants to apply the Mikhailov's reduction theory [15] to the system $L$ the element $J$ must obey some restrictions showing that $J$ cannot be always real. However, the case when $J$ is not real turned out to be more complicated. For $\mathfrak{g}=\mathfrak{s l}(n, \mathbb{C})$ in the simplest representation it was considered by Caudrey, Beals and Coifman [2-5]. The reason complex $J$ case is more involved is that the spectrum of the corresponding linear problem is quite different from the real $J$ case. We call the linear system with complex $J$ the Caudrey-Beals-Coifman (CBC) system. We considered this system for arbitrary simple Lie algebra $\mathfrak{g}$ in arbitrary finite dimensional faithful representation in [11] and concentrated on the adjoint representation. The point is that this representation is essential in the so-called $\Lambda$-operator approach (or generating operator approach) to the soliton equations related to $L$.

The generating operator approach to the NLEEs related to $L$ originates from the paper of Ablowitz, Kaup, Newell and Segur [1] which treats the ZS system. It has been generalized for the case of arbitrary semisimple algebra and real $J$ in [8]. The method could also be generalized for another systems, see [9] and the references therein , and the calculation of the Generating Operators becomes an important issue, see [14] about this issue. The presence of Mikhailov-type reductions [15] changes significantly the theory of the recursion operators, both regarding their spectral properties [17] and their geometric properties, [18]. Thus it becomes important to go carefully through the proofs and formulae in order to understand what should be changed in the general theory. Somewhat surprisingly we discovered that in the generating operator approach there are some points regarding the conservation laws that remained in shadow when the generalization from the the ZS system 
to GZS system and next to CBC system was made and and of course they should be clarified. This is what we intend to do in the present article.

\section{Fundamental Solutions to the CBC System}

In order to introduce the generating operator theory we need some of the spectral properties of the CBC system so we first discuss shortly the fundamental solutions of $L \psi=0$. The missing details can be found in [11].

It turns out that it is convenient to study not the equation $L \psi=0$, see (3), but the equation satisfied by the function $m(x, \lambda)=\psi(x, \lambda) \exp \mathrm{i} \lambda J x$. Assume that in the space $V$ we have fixed finite-dimensional irreducible representation of the simple Lie algebra $\mathfrak{g}$ with root system $\Delta$. This equation together with an asymptotic condition for $m$ has the form

$$
\mathrm{i} \partial_{x} m+q(x) m-\lambda J m+\lambda m J=0, \quad \lim _{x \rightarrow-\infty} m=\mathbf{1}_{V} .
$$

We are looking for bounded solutions and in that case one can prove that if $\lambda$ does not belong to the bunch of straight lines

$$
\Sigma=\{\lambda ; \operatorname{Im}(\lambda \alpha(J))=0, \quad \alpha \in \Delta\}
$$

the solution $m(x, \lambda)$ (if it exists) is unique.

Now we outline the construction of the fundamental analytic solutions to (4), see [11]. Suppose we denote by Greek letters $\alpha, \beta, \gamma \ldots$ the weights of the representation of $\mathfrak{g}$ in $V$ and the set of all weights by $\Gamma$. For $\alpha, \beta \in \Gamma$, consider the lines $\Sigma_{\alpha, \beta}=\{\lambda ; \operatorname{Im} \lambda(\alpha-\beta)(J)=0\},(\alpha-\beta)(J) \neq 0$. It can be shown that $\Sigma=\bigcup_{\alpha, \beta \in \Gamma} \Sigma_{\alpha, \beta}$ where $\Sigma$ is as in (5). Then the connected components of $\mathbb{C} \backslash \Sigma$ in typical representation are sectors in the $\lambda$-plain. In every such sector either $\operatorname{Im}[\lambda(\alpha-\beta)(J)]$ is identically zero (if $\alpha=\beta$ ) or it does not change sign. We shall denote these sectors by $\Omega_{\nu}$ ordering them anti-clockwise. Clearly $\nu$ takes values from 1 to some even number $2 M$ and it is natural to understand $\nu$ modulo $2 M$. We have $\mathbb{C} \backslash \Sigma=\bigcup_{\nu=1}^{2 M} \Omega_{\nu}, \Omega_{\nu} \bigcap \Omega_{\mu}=\emptyset$ if $\nu \neq \mu$. In the $\nu$-th sector we introduce the ordering: $\alpha \geq_{\nu} \beta$ iff $\operatorname{Im} \lambda(\alpha-\beta)(J) \geq 0$ and $\alpha>_{\nu} \beta$ iff $\operatorname{Im} \lambda(\alpha-\beta)(J)>0$. Then it can be shown that using the ordering in $\Omega_{\nu}, \nu=1,2, \ldots, 2 M$ one can introduce in each $\Omega_{\nu}$ a system of integral equations equivalent to (4). Its analysis permits to establish that under quite general assumptions for the potential $q(x)$ (for the details see [11]) in each $\Omega_{\nu}$ exists unique solution $m_{\nu}$ which is analytic in $\lambda \in \Omega_{\nu}$ (except some poles) and allowing extension by continuity to the boundary of $\Omega_{\nu}$, that is to the rays $L_{\nu}$ and $L_{\nu+1}$, see [20] for the GZS system case, [2,5] for the $\mathfrak{s l}(n)$ case and [11] for the general case. The continuous spectrum of the problem lies on the rays $L_{\nu}$ and the poles we mentioned define the discrete spectrum. 
In particular, for potentials with small $L^{1}$ norm we do not have discrete spectrum. In the present work however we shall not write the discrete spectrum contributions and then one might say that we limit ourselves to the case of small potentials.

Knowing the solutions $m_{\nu}(x, \lambda)$ one can construct the fundamental solutions (FAS) $\chi_{\nu}(x, \lambda)$ of the CBC system setting $\chi_{\nu}(x, \lambda)=m_{\nu}(x, \lambda) \mathrm{e}^{\mathrm{i} J x \lambda}$ and using them to build the spectral theory of the operator $L$ in any faithful representation of $\mathfrak{g}$. Since the Lax reprsentation in fact uses the adjoint representation it is of particular interest to us.

\section{Generating Operator Approach to the Soliton Equations Associated with the CBC System}

\subsection{Expansions Over the Adjoint Solutions for the CBC System}

We need to fix first some notation. First, because of the superscripts we must use the formulae that become cumbersome when we need to write an inverse element so in the future we shall use 'hat' in order to denote the inverse. Further, if we have a function $f(\lambda)$ that is section analytic on the sectors $\Omega_{\nu}$ and in each sector $\Omega_{\nu}$ it allows extension by continuity to the boundary of the sector, then for $\lambda$ on the ray $L_{\nu}$ we shall denote the restriction of $f$ in the sector $\Omega_{\nu}$ by $f_{\nu}(\lambda)$, and for $\lambda$ belonging to the ray $L_{\nu}$ the limit from $\Omega_{\nu}$ by $f_{\nu}^{+}(\lambda)$ and from $\Omega_{\nu-1}$ by $f_{\nu}^{-}(\lambda)$. Next, let us define

$$
\begin{aligned}
& \delta_{\nu}^{ \pm}=\Delta_{\nu}^{ \pm} \cap \delta_{\nu}, \quad \delta_{\nu}=\left\{\alpha \in \Delta ; \operatorname{Im}(\lambda \alpha(J))=0 \text { for } \lambda \in L_{\nu}\right\} \\
& \mathfrak{g}_{\nu} \text { - the semisimple algebra with root system } \delta_{\nu} \\
& \pi_{\nu} \text { - the system of simple roots for } \mathfrak{g}_{\nu} .
\end{aligned}
$$

Now we are ready to describe the jumps of the solutions $m(x, \lambda)$ on the rays $L_{\nu}$. For $\lambda \in L_{\nu}$ they are given by

$$
\begin{aligned}
& m_{\nu}^{+}(x, \lambda)=m_{\nu}^{-}(x, \lambda) \mathrm{e}^{-\mathrm{i} J \lambda x} g_{\nu}(\lambda) \mathrm{e}^{\mathrm{i} J \lambda x} \\
& g_{\nu}(\lambda)=\hat{S}_{\nu}^{-}(\lambda) S_{\nu}^{+}(\lambda)=\hat{D}_{\nu}^{-}(\lambda) \hat{T}_{\nu}^{+}(\lambda) T_{\nu}^{-}(\lambda) D_{\nu}^{+}(\lambda) .
\end{aligned}
$$

Here $S_{\nu}^{ \pm}(\lambda), T_{\nu}^{ \pm}(\lambda), D_{\nu}^{ \pm}(\lambda)$ have the form

$$
\begin{aligned}
S_{\nu}^{ \pm}(\lambda) & =\exp \sum_{\alpha \in \delta_{\nu}^{+}} s_{\nu, \alpha}^{ \pm}(\lambda) E_{ \pm \alpha}, \quad T_{\nu}^{ \pm}(\lambda)=\exp \sum_{\alpha \in \delta_{\nu}^{+}} t_{\nu, \alpha}^{ \pm}(\lambda) E_{ \pm \alpha} \\
D_{\nu, \alpha}^{ \pm}(\lambda) & =\exp \sum_{\alpha \in \pi_{\nu}} d_{\nu, \alpha}^{ \pm}(\lambda) H_{\alpha}
\end{aligned}
$$


so that $S_{\nu}^{ \pm}, T_{\nu}^{ \pm}, D_{\nu}^{ \pm}$belong to the subgroup $G_{\nu}$ with Lie algebra $\mathfrak{g}_{\nu}$. They are defined by the behaviour of $m_{\nu}^{ \pm}(x, \lambda)$ when $x \rightarrow \pm \infty$, see [11], Theorem 3.3

$$
S_{\nu}^{ \pm}(\lambda)=\lim _{x \rightarrow-\infty} \mathrm{e}^{\mathrm{i} J \lambda x} \chi_{\nu}^{ \pm}(x, \lambda), \quad T_{\nu}^{\mp}(\lambda) D_{\nu}^{ \pm}(\lambda)=\lim _{x \rightarrow+\infty} \mathrm{e}^{\mathrm{i} J \lambda x} \chi_{\nu}^{ \pm}(x, \lambda) .
$$

Define in each $\Omega_{\nu}$ the functions

$$
e_{\alpha}^{\nu}(x, \lambda)=\pi_{0}\left(\chi_{\nu}(x, \lambda) E_{\alpha} \chi_{\nu}^{-1}(x, \lambda)\right), \quad \lambda \in \Omega_{\nu} .
$$

According to our previous agreement for $\lambda \in L_{\nu}$ we write $m_{\nu}^{+}(x, \lambda)$ and $\chi_{\nu}^{+}(x, \lambda)$ if the solution is extended from the sector $\Omega_{\nu}$ and $m_{\nu}^{-}(x, \lambda)\left(\chi_{\nu}^{-}(x, \lambda)\right)$ if it is extended from $\Omega_{\nu-1}$. Analogously, we write $e_{\alpha}^{(-; \nu)}(x, \lambda)$ if the solution is extended from the sector $\Omega_{\nu-1}$ and $e_{\alpha}^{(+; \nu)}(x, \lambda)$ if the solution is extended from the sector $\Omega_{\nu}$. Both the sets

$$
\begin{aligned}
& E_{\rho}=\bigcup_{\nu=1}^{2 M}\left\{e_{\alpha}^{(+; \nu)}(x, \lambda), e_{-\alpha}^{(-; \nu)}(x, \lambda) ; \alpha \in \delta_{\nu}^{+}, \lambda \in L_{\nu}\right\} \\
& E_{\sigma}=\bigcup_{\nu=1}^{2 M}\left\{e_{-\alpha}^{(+; \nu)}(x, \lambda), e_{\alpha}^{(-; \nu)}(x, \lambda) ; \alpha \in \delta_{\nu}^{+}, \lambda \in L_{\nu}\right\}
\end{aligned}
$$

are complete in the space of $L^{1}$-integrable function $h: \mathbb{R} \mapsto \overline{\mathfrak{g}}$ in the same sense as the exponents are. (For the form of the completeness relations see [11] or for the form that we shall use see [17].) The above functions are called adjoint solutions or Generalized Exponents. Using Theorem 3.2 from [11] one can prove that

$$
\begin{array}{lll}
\left(\Lambda_{-}-\lambda\right) e_{\alpha}^{(+; \nu)}=0, & \left(\Lambda_{-}-\lambda\right) e_{-\alpha}^{(-; \nu)}=0, & \alpha \in \delta_{\nu}^{+} \\
\left(\Lambda_{+}-\lambda\right) e_{-\alpha}^{(+; \nu)}=0, & \left(\Lambda_{+}-\lambda\right) e_{\alpha}^{(-; \nu)}=0, & \alpha \in \delta_{\nu}^{+}
\end{array}
$$

where the operators $\Lambda_{ \pm}$are given by

$\Lambda_{ \pm}(X(x))=\operatorname{ad}_{J}^{-1}\left(\mathrm{i} \partial_{x} X+\pi_{0}[q, X]+\operatorname{iad}_{q} \int_{ \pm \infty}^{x}\left(\mathrm{id}-\pi_{0}\right)[q(y), X(y)] \mathrm{d} y\right)$.

The above operators are the famous generating, recursion or $\Lambda$-operators related to the CBC system, see $[9,11]$. To see their role consider the expansions over the Generalized Exponents of the potential $q(x)$ and its variation $\delta q(x)$. More specifically, let $B$ is constant element from $\mathfrak{h}$. Then for the expansions over $E_{\rho}$ (there are analogous expansions over $E_{\sigma}$ but we do not write them) we have

$$
\operatorname{ad}_{J}^{-1}[B, q](x)=\frac{\mathrm{i}}{2 \pi} \sum_{\nu=1}^{2 M} \int_{L_{\nu}} \sum_{\alpha \in \delta_{\nu}^{+}}\left(\rho_{\nu ; B,-\alpha}^{+} e_{\alpha}^{(+; \nu)}-\rho_{\nu ; B, \alpha}^{-} e_{-\alpha}^{(-; \nu)}\right) \mathrm{d} \lambda
$$


where

$$
\rho_{\nu ; B, \mp \alpha}^{ \pm} \equiv \mathrm{i} \int_{-\infty}^{+\infty}\left\langle[q, B], e_{\mp \alpha}^{( \pm ; \nu)}\right\rangle \mathrm{d} x=\left\langle\hat{S}_{\nu}^{ \pm} B S_{\nu}^{ \pm}, E_{\mp \alpha}\right\rangle .
$$

The expansions for $\operatorname{ad}_{J}^{-1} \delta q$ run as follows

$$
\operatorname{ad}_{J}^{-1} \delta q(x)=\frac{\mathrm{i}}{2 \pi} \sum_{\nu=1}^{2 M} \int_{L_{\nu}} \sum_{\alpha \in \delta_{\nu}^{+}}\left(\delta \rho_{\nu ;-\alpha}^{+} e_{\alpha}^{(+; \nu)}-\delta \rho_{\nu ; \alpha}^{-} e_{-\alpha}^{(-; \nu)}\right) \mathrm{d} \lambda
$$

where

$$
\delta \rho_{\nu ; \mp \alpha}^{ \pm}(\lambda) \equiv-\mathrm{i} \int_{-\infty}^{+\infty}\left\langle\delta q, e_{\mp \alpha}^{( \pm ; \nu)}\right\rangle \mathrm{d} x=\left\langle\hat{S}_{\nu}^{ \pm} \delta S_{\nu}^{ \pm}, E_{\mp \alpha}\right\rangle(\lambda) .
$$

Let us introduce now a set of scattering data

$$
\mathcal{T}_{\rho, B}=\bigcup_{\nu=1}^{2 M}\left\{\rho_{\nu ; B,-\alpha}^{+}(\lambda), \rho_{\nu ; B, \alpha}^{-}(\lambda) ; \alpha \in \Delta_{\nu}^{+}, \lambda \in L_{\nu}\right\} .
$$

The formulae (16) and (18) show that the mapping from the potential function $q(x)$ to the scattering data $\mathcal{T}_{\rho}$ can be regarded as generalized Fourier transform. Then indeed (17) play the role of the inverse Fourier transform formulae. The Generalized exponents play the same role as the usual exponents in the Fourier transform and the operators $\Lambda_{ \pm}$play the role the operator $i \partial_{x}$ plays for the usual Fourier transform.

\section{The NLEEs Related to the CBC System}

\subsection{General Description}

The NLEEs associated with the linear problem $L$ (and integrable through some kind of inverse scattering technique for $L$ ) are most easily found if we adopt the approach based on the expansions (16), (19). Indeed, consider the equations having the form

$$
\sum_{k=1}^{r} f_{k}\left(\Lambda_{ \pm}\right) \operatorname{ad}_{J}^{-1}\left[H_{k}, q\right]+\operatorname{iad}_{J}^{-1} q_{t}=0
$$

where $f(\lambda)=\sum_{k=1}^{r} f_{k}(\lambda) H_{k}$. Here $\left\{H_{k}\right\}_{k=1}^{r}$ is the part of the Cartan-Weil basis giving a basis in $\mathfrak{h}$ and $f_{k}(\lambda)$ are polynomials in $\lambda$ and $\lambda^{-1} \cdot f(\lambda)$ is known as the 
dispersion law of the corresponding NLEE. Expanding through the Generalized Exponents it could be written also in the following equivalent form

$$
\mathrm{i} \frac{\mathrm{d} \rho_{\nu ; \mp \alpha}^{ \pm}}{\mathrm{d} t}+\sum_{k=1}^{r} f_{k}(\lambda) \rho_{\nu ; H_{k}, \mp \alpha}^{ \pm}(\lambda, t)=0, \quad 1 \leq \nu \leq 2 M
$$

where of course for the functions with index $\nu$ the argument $\lambda$ belongs to $L_{\nu}$. Naturally, this is in accordance with the fact that the equations we are speaking about have a Lax representation of the type $[L, A]=0$. In terms of the scattering data factors $S_{\nu}^{ \pm}, T_{\nu}^{ \pm}, D_{\nu}^{ \pm}$the equation (21) is written as

$$
\mathrm{i} \frac{\mathrm{d} S_{\nu}^{ \pm}}{\mathrm{d} t}+\left[f(\lambda), S_{\nu}^{ \pm}\right]=0, \quad \mathrm{i} \frac{\mathrm{d} T_{\nu}^{ \pm}}{\mathrm{d} t}+\left[f(\lambda), T_{\nu}^{ \pm}\right]=0, \quad \mathrm{i} \frac{\mathrm{d} D_{\nu}^{ \pm}}{\mathrm{d} t}=0 .
$$

This shows that $D_{\nu}^{ \pm}$are not changed by the evolution and then naturally they are related to the conservation laws for (21). In fact some functions obtained through them, see (10) below, are generating functions for the conservation laws.

\subsection{Conservation Laws}

It is well known, see for example [6], that the evolution equations (21) have $r=$ rankg series of conservation laws. Below we present the formulae for the conservation laws obtained through the theory of recursion operators. Their advantage is that they give a formula for the conservation laws while the conservation laws obtained via another approaches are constructed by recurrent procedures. Having a formula is an advantage, it gives us the possibility to describe which of them trivialize if we have reductions, see [12].

We introduce first some properties of the functions $h_{\nu, H}(x, \lambda)=\chi_{\nu} H \hat{\chi}_{\nu}(x, \lambda)$, $H \in \mathfrak{h}, x \in \mathbb{R}, \lambda \in \Omega_{\nu}$ and more precisely of their projections $h_{\nu, H}^{a}(x, \lambda)=$ $\pi_{0} h_{\nu, H}(x, \lambda)$ as well as for the corresponding extensions $h_{\nu, H}^{ \pm}(x, \lambda), h_{\nu, H}^{ \pm a}(x, \lambda)$ of these functions to the rays $L_{\nu}$. Here of course $\chi_{\nu}(x, \lambda)$ is a FAS to the CBC system analytic in the sector $\Omega_{\nu}$.

\subsubsection{Properties of the Adjoint Solutions $h_{\nu, H}$}

It is not hard to prove the following results which are important ingredients for our construction.

Proposition 1. Let $H \in \mathfrak{h}$ be an arbitrary element from the Cartan subalgebra. Let us consider $h_{\nu, H}(x, \lambda)=\chi_{\nu}(x, \lambda) H \chi_{\nu}^{-1}(x, \lambda)$. Suppose the derivatives of the 
potential $q(x)$ up to the order $N$ belong to the class $L^{1}(\mathbb{R})$. Suppose also that $S_{\nu}$ is proper open sub-sector of $\Omega_{\nu}$. Then for $\lambda$ tending to infinity but remaining in $S_{\nu}$ we have the following asymptotic formulae which hold uniformly in $x$

$$
h_{\nu, H}=H+\sum_{k=1}^{N} \lambda^{-k}\left(\Lambda_{-}^{k-1} q_{H}+\mathrm{iI}_{-}\left(\Lambda_{-}^{k-1}\right) q_{H}\right)+\mathrm{o}\left(\lambda^{-N}\right)
$$

where we have used the notation

$$
q_{H} \equiv \operatorname{ad}_{J}^{-1}[q, H], \quad \mathrm{I}_{ \pm} f \equiv \int_{ \pm \infty}^{x}\left(\mathrm{id}-\pi_{0}\right) \operatorname{ad}_{q(y)} f(y) \mathrm{d} y .
$$

In the above $f(x)$ is differentiable, absolutely integrable on the line, taking values in $\overline{\mathfrak{g}}$ and $\Lambda_{-}$is the operator we introduced in (15). In what follows up to the end of this subsection we shall assume that the potential $q(x)$ is a Schwartz-type function. Then the asymptotic formula can be written for arbitrary $N$ and all the expressions we write make sense.

Proposition 2. The following integral representations hold

1. For $\lambda \in \Omega_{\nu}$ we have

$$
h_{\nu, H}^{a}(x, \lambda)=\frac{\mathrm{i}}{2 \pi} \sum_{\eta=1}^{2 M} \int_{L_{\eta}} \frac{h_{\eta, H}^{-a}(x, \mu)-h_{\eta, H}^{+a}(x, \mu)}{\mu-\lambda} \mathrm{d} \mu .
$$

2. For $\lambda$ on the ray $L_{\nu}$ and $\lambda \neq 0$ we have

$$
\frac{1}{2}\left(h_{\nu, H}^{-a}(x, \lambda)-h_{\nu, H}^{+a}(x, \lambda)\right)=\frac{\mathrm{i}}{2 \pi} \sum_{\eta=1}^{2 M} \mathrm{p} . \mathrm{v} \cdot \int_{L_{\eta}} \frac{h_{\eta, H}^{-a}(x, \mu)-h_{\eta, H}^{+a}(x, \mu)}{\mu-\lambda} \mathrm{d} \mu .
$$

Of course the principle value is necessary to be taken only on the ray $L_{\nu}$ but in the way the things are now written (27) becomes more symmetric.

The functions $h_{\nu, H}^{a}$ are closely related to the expansions of the potential $q(x)$. Indeed, if we recall the definition of the coefficients $\rho_{H, \alpha}^{ \pm}$introduced earlier, see (17), then we easily have

$$
h_{\nu, H}^{-a}-h_{\nu, H}^{+a}=\sum_{\alpha \in \delta_{\nu}^{+}}\left(\rho_{\nu ; H,-\alpha}^{+}(\lambda) e_{\alpha}^{(+; \nu)}-\rho_{\nu ; H, \alpha}^{-}(\lambda) e_{-\alpha}^{(-; \nu)}\right) .
$$


Using the expansion (16) and the above expressions we get

$$
\frac{\mathrm{i}}{2 \pi} \sum_{\nu=1}^{2 M} \int_{L_{\nu}}\left(h_{\nu, H}^{-a}(x, \lambda)-h_{\nu, H}^{+a}(x, \lambda)\right) d \lambda=\operatorname{ad}_{J}^{-1}[H, q](x)
$$

and then for $N=1,2, \ldots$

$$
\Lambda_{ \pm}^{N}\left(h_{\nu, H}^{-a}(x, \lambda)-h_{\nu, H}^{+a}(x, \lambda)\right)=\lambda^{N}\left(h_{\nu, H}^{-a}(x, \lambda)-h_{\nu, H}^{+a}(x, \lambda)\right) .
$$

Combining the integral representations (26) with (29) from here we obtain that for $\lambda \in \Omega_{\nu}$

$$
h_{\nu, H}^{a}=\left(\Lambda_{ \pm}-\lambda\right)^{-1} \operatorname{ad}_{J}^{-1}[H, q] .
$$

This of course is in accordance with the fact the asymptotic expansion (24).

\subsubsection{Conservation Laws. The Generating Functions}

The series of conservation laws for the NLEEs related to CBC system can be found in complete analogy to the case when $J$ is real. Roughly speaking, the functions $D_{\nu}^{ \pm}(\lambda)$ (or their 'logarithms') are generating functions for the conservation laws of the above NLEEs. More precisely, see [11], we have the following situation. For $\lambda \in \Omega_{\nu}$ define

$$
\begin{aligned}
D_{\nu, j}(\lambda) & =\lim _{x \rightarrow+\infty}\left\langle\omega_{\nu, j}\left|\mathrm{e}^{\mathrm{i} \lambda J x} m_{\nu}(\lambda, x) \mathrm{e}^{-\mathrm{i} \lambda J x}\right| \omega_{\nu, j}\right\rangle=\lim _{x \rightarrow+\infty}\left\langle\omega_{\nu, j}\left|m_{\nu}(\lambda . x)\right| \omega_{\nu, j}\right\rangle \\
j & =1,2, \ldots r=\operatorname{rankg}, \quad \nu=1,2, \ldots 2 M .
\end{aligned}
$$

These functions can be extended to the boundaries of the sectors and for $\lambda \in L_{\nu}$ we have $D_{\nu+1, j}(\lambda)=D_{\nu, j}^{+}(\lambda), D_{\nu, j}(\lambda)=D_{\nu, j}^{-}(\lambda)$. In addition, $\lim _{\lambda \rightarrow \infty} D_{\nu, j}=1$. For $j=1,2, \ldots r=\operatorname{rankg}, \nu=1,2, \ldots 2 M$ define

$$
d_{\nu, j}(\lambda)=\log D_{\nu, j}(\lambda) .
$$

The functions $d_{\nu, j}(\lambda)$ are analytic in $\Omega_{\nu}$ and $\lim _{\lambda \rightarrow \infty} d_{\nu, j}(\lambda)=0$. If one considers them in any sub-sector $S_{\nu}$ of $\Omega_{\nu}$ one has the asymptotic expansions

$$
d_{\nu, j}(\lambda)=\sum_{s=1}^{\infty} d_{\nu, j, s} \lambda^{-s}, \quad \lambda \in S_{\nu}, \quad|\lambda|>>1
$$

and $d_{\nu, j, s}$ are then the required conservation laws. It turns out that it is easier to work with some linear combinations of the functions $d_{\nu, j}(\lambda)$. Namely, for $H \in \mathfrak{h}$ 
define $d_{\nu, H}(\lambda)=\sum_{\alpha \in \pi_{\nu}} d_{\nu, \alpha}(\lambda)\left\langle H_{\alpha}, H\right\rangle$. In any sub-sector $S_{\nu}$ of $\Omega_{\nu}$ we have the asymptotic

$$
d_{\nu, H}(\lambda)=\sum_{k=1}^{\infty} d_{H, k} \lambda^{-k}, \quad|\lambda|>>1 .
$$

One can prove that actually the coefficients in the asymptotic expansion do not depend on the sector so in the right hand side we are not writing the index $\nu$. The usual way to obtain the coefficients $d_{H, k}$ is to use some Wronskian-type relations which we introduce below in (37) and (44). To this end, denote the $\lambda$-derivative of $\chi_{\nu}^{ \pm}$by $\dot{\chi}_{\nu}^{ \pm}$where $\chi_{\nu}^{ \pm}$are the FAS of the CBC system we introduced earlier.. Then one easily checks that

$$
\frac{\mathrm{d} \dot{\chi}_{\nu}^{ \pm}}{\mathrm{d} x}(x, \lambda)=(\lambda J-q(x)) \dot{\chi}_{\nu}^{ \pm}+J \chi_{\nu}^{ \pm}
$$

as the $\lambda$-derivative of $\chi_{\nu}(x, \lambda)$ allows extensions to $L_{\nu-1}$ and $\left.L_{\nu}\right)$. As a result we have the following Wronskian-type relation

$$
\left.\left(\mathrm{i} \hat{\chi}_{\nu}^{ \pm} \frac{\mathrm{d} \chi_{\nu}^{ \pm}}{\mathrm{d} \lambda}(x, \lambda)-J x\right)\right|_{-\infty} ^{+\infty}=\int_{-\infty}^{+\infty}\left(\hat{\chi}_{\nu}^{ \pm} J \chi_{\nu}^{ \pm}(x, \lambda)-J\right) \mathrm{d} x, \quad \lambda \in L_{\nu}
$$

Now, for the inner product of the left hand side of (37) with $H \in \mathfrak{h}$ we get

$$
\mathrm{i}\left\langle\hat{D}_{\nu}^{ \pm} \dot{D}_{\nu}^{ \pm}, H\right\rangle=\mathrm{i} \sum_{\alpha \in \pi_{\nu}} \dot{d}_{\nu, \alpha}^{ \pm}\left\langle H_{\alpha}, H\right\rangle \equiv \mathrm{i} \dot{d}_{\nu, H}^{ \pm}
$$

where as usual with superscripts ${ }^{ \pm}$are denoted the extensions on $L_{\nu}$ from the left and the right. In the sectors we have

$$
\mathrm{i}\left\langle\hat{D}_{\nu} \dot{D}_{\nu}, H\right\rangle=\mathrm{i} \sum_{\alpha \in \pi_{\nu}} \dot{d}_{\nu, \alpha}\left\langle H_{\alpha}, H\right\rangle \equiv \mathrm{i} \dot{d}_{\nu, H}
$$

Next, taking inner product of the right side of (37) with $H \in \mathfrak{h}$, we obtain

$$
\int_{-\infty}^{+\infty}\left\langle\hat{\chi}_{\nu}^{ \pm} J \chi_{\nu}^{ \pm}-J, H\right\rangle \mathrm{d} x=-\mathrm{i} \int_{-\infty}^{+\infty} \int_{-\infty}^{x}\left\langle H, \mathrm{i} \frac{\partial}{\partial y} \hat{\chi}_{\nu}^{ \pm} J \chi_{\nu}^{ \pm}(y, \lambda)\right\rangle \mathrm{d} y \mathrm{~d} x .
$$

The integrand in the left hand side of the above equation tends fast enough to zero so the integral exists.) It is easy to see that this equals

$$
\mathrm{i} \int_{-\infty}^{+\infty} \int_{-\infty}^{x}\left\langle H, \hat{\chi}_{\nu}^{ \pm}[J, q] \chi_{\nu}^{ \pm}(y, \lambda)\right\rangle \mathrm{d} y \mathrm{~d} x=\mathrm{i} \int_{-\infty}^{+\infty} \int_{-\infty}^{x}\left\langle h_{\nu, H}^{a \pm}(y, \lambda),[J, q](y)\right\rangle \mathrm{d} y \mathrm{~d} x
$$


where $h_{\nu, H}^{a \pm}(y, \lambda)=\pi_{0} \chi_{\nu}^{ \pm}(y, \lambda) H \hat{\chi}_{\nu}^{ \pm}, H \in \mathfrak{h}$. Thus in $\Omega_{\nu}$ we obtain

$$
\dot{d}_{\nu, H}(\lambda)=\int_{-\infty}^{+\infty} \int_{-\infty}^{x}\left\langle h_{\nu, H}^{a},[J, q]\right\rangle(y, \lambda) \mathrm{d} y \mathrm{~d} x .
$$

Now taking into account the asymptotic expansion for $h_{\nu, H}^{a}$ we get

$$
d_{H, s}=\frac{1}{s} \int_{-\infty}^{+\infty} \int_{-\infty}^{x}\left\langle[J, q], \Lambda_{ \pm}^{s} \operatorname{ad}_{J}^{-1}[H, q]\right\rangle \mathrm{d} y \mathrm{~d} x, \quad s=1,2, \ldots
$$

In a similar way, using the Wronskian type relation

$$
\left.\mathrm{i}\left\langle\hat{\chi}_{\nu}^{ \pm} \delta \chi_{\nu}^{ \pm}, H\right\rangle\right|_{-\infty} ^{+\infty}=-\int_{-\infty}^{+\infty}\left\langle\hat{\chi}_{\nu}^{ \pm} \delta \chi_{\nu}^{ \pm}, H\right\rangle \mathrm{d} x
$$

for $H \in \mathfrak{h}$, we get

$$
\delta d_{H, s}=-\mathrm{i} \int_{-\infty}^{+\infty}\left\langle\delta q, \Lambda_{ \pm}^{s-1} \operatorname{ad}_{J}^{-1}[H, q]\right\rangle \mathrm{d} x, \quad s=1,2, \ldots
$$

The above relation permits to find another, frequently more convenient expression, for the conservation laws, see [12]

$$
d_{H, s}=-\mathrm{i} \int_{-\infty}^{+\infty} \int_{0}^{1}\left\langle q,\left.\Lambda_{ \pm}^{s-1}\right|_{(\zeta q)} \operatorname{ad}_{J}^{-1}[H, \zeta q]\right\rangle \mathrm{d} \zeta \mathrm{d} x .
$$

\section{Locality of the Hierarchy of NLEEs Related to the CBC System and Their Conservation Laws}

In this section we treat the questions of the locality of the NLEEs related to the CBC system and their conservation laws, proving also that the conservation laws are in involution with respect to the hierarchy of symplectic structures. The idea of the proof we present has been used in [10] for the case of the classical ZS system but because the Cartan subalgebra for the classical ZS system is one-dimensional the proof is much simpler, in fact it requires only the identity in Lemma 5 below.

Proposition 3. If for arbitrary $H, W \in \mathfrak{h}$ the expression $\left\langle J,\left[q_{H}, \Lambda_{-}^{N} q_{W}\right]\right\rangle$ is $x$ derivative of local function on $q, q_{x}, \ldots$ then the expression $\Lambda_{-}^{N+1} q_{F}$ for every $F \in \mathfrak{h}$ is also a local function. 
Proof: Indeed

$$
\Lambda_{-} \Lambda_{-}^{N} q_{F}=\operatorname{iad}_{J}^{-1} \partial_{x} \Lambda_{-}^{N} q_{F}+\operatorname{ad}_{J}^{-1}\left[q, \pi_{0} \Lambda_{-}^{N} q_{F}\right]+\operatorname{iad}_{J}^{-1} \operatorname{ad}_{q} \mathrm{I}_{-} \Lambda_{-}^{N} q_{F} .
$$

The expression in the integrand of $\mathrm{I}_{-} \Lambda_{-}^{N} q_{F}$ can be cast into the following form

$$
\left(\mathrm{id}-\pi_{0}\right)\left[q, \Lambda_{-}^{N} q_{F}\right]=\sum_{s=1}^{r} H_{s}\left\langle H^{s},\left[q, \Lambda_{-}^{N} q_{F}\right]\right\rangle
$$

where $\left\{H_{s}\right\}_{s=1}^{r},\left\{H^{s}\right\}_{s=1}^{r}$ are two bi-orthogonal bases of $\mathfrak{h}$. By assumption I_ $\Lambda_{-}^{N} q_{F}$ is local so the expression $\Lambda_{-}^{N+1} q_{F}$ is also local.

Corollary 4. In all the above formulae one can put $\Lambda_{+}, I_{+}$instead of $\Lambda_{-}, I_{-}$.

Proof: Indeed, for any $N=1,2, \ldots$ we have

$\int_{-\infty}^{+\infty}\left(\mathrm{id}-\pi_{0}\right) \Lambda_{-}^{N} q_{F} \mathrm{~d} x=0$ so since $\mathrm{I}_{-} \Lambda_{-}^{N} q_{F}=\mathrm{I}_{+} \Lambda_{-}^{N} q_{F}+\int_{-\infty}^{+\infty}\left(\mathrm{id}-\pi_{0}\right) \Lambda_{-}^{N} q_{F} \mathrm{~d} x$

so we get $\mathrm{I}_{-} \Lambda_{-}^{N} q_{F}=\mathrm{I}_{+} \Lambda_{-}^{N} q_{F}$ for arbitrary natural $N$. Hence $N \Lambda_{-}^{N} q_{F}=\Lambda_{+}^{N} q_{F}$.

Thus we need to prove that $\left\langle J,\left[q_{H}, \Lambda_{-}^{N} q_{W}\right]\right\rangle$ is a total derivative of a local function on $q, q_{x}, \ldots$. For this we need some relations and identities that we introduce in the next lemmas.

Lemma 5. For $H, W \in \mathfrak{h}$ and integer $N$

$$
\left\langle J,\left[q_{H}, \Lambda_{-}^{N} q_{W}\right]\right\rangle-\left\langle J,\left[\Lambda_{-}^{N} q_{H}, q_{W}\right]\right\rangle=\mathrm{i} \partial_{x} \mathfrak{F}_{1}(H, W ; N-1)
$$

where $\mathfrak{F}_{1}(H, W ; N-1)$ is a polynomial with constant coefficients on the quantities $\left\langle\mathrm{I}_{-} \Lambda_{-}^{k} q_{H}, \mathrm{I}_{-} \Lambda_{-}^{s} q_{W}\right\rangle$ and $\left\langle\Lambda_{-}^{k} q_{H}, \Lambda_{-}^{s} q_{W}\right\rangle, 0 \leq s, k \leq N-1$.

Proof: From the differential equation for $h_{\nu, H}$ it is readily seen that for $H, W \in \mathfrak{h}$, $\lambda \in \Omega_{\nu}, \mu \in \Omega_{\eta}$ we have the following relations

$$
\mathrm{i} \partial_{x}\left\langle h_{\nu, H}(x, \lambda), h_{W, \eta}(x, \mu)\right\rangle=(\lambda-\mu)\left\langle J,\left[h_{H, \nu}(x, \lambda), h_{W, \eta}(x, \mu)\right]\right\rangle .
$$

For big $\lambda, \mu$ we can insert the asymptotic formulae we obtained in Proposition 1 and as the asymptotic are uniform in $x$ we can differentiate. Comparing then the coefficients of the power series we obtain the following identities

$$
\begin{aligned}
\left\langle J,\left[\Lambda_{-}^{k-1} q_{H}, \Lambda_{-}^{s} q_{W}\right]\right\rangle-\left\langle J,\left[\Lambda_{-}^{k} q_{H}, \Lambda_{-}^{s-1} q_{W}\right]\right\rangle \\
\quad=\mathrm{i} \partial_{x}\left(\left\langle\mathrm{I}_{-} \Lambda_{-}^{k-1} q_{H}, \mathrm{I}_{-} \Lambda_{-}^{s-1} q_{W}\right\rangle-\left\langle\Lambda_{-}^{k-1} q_{H}, \Lambda_{-}^{s-1} q_{W}\right\rangle\right) .
\end{aligned}
$$


Using them one easily gets

$$
\begin{aligned}
\langle J, & {\left.\left[q_{H}, \Lambda_{-}^{N} q_{W}\right]\right\rangle-\left\langle J,\left[\Lambda_{-}^{N} q_{H}, q_{W}\right]\right\rangle } \\
& =\mathrm{i} \partial_{x}\left\{\sum_{k=0}^{N-1}\left(\left\langle\mathrm{I}_{-} \Lambda_{-}^{k} q_{H}, \mathrm{I}_{-} \Lambda_{-}^{N-k-1} q_{W}\right\rangle-\left\langle\Lambda_{-}^{k} q_{H}, \Lambda_{-}^{N-k-1} q_{W}\right\rangle\right)\right\} .
\end{aligned}
$$

The Lemma is proved.

Now we need another identity. It is easy to see that for $\lambda \in \Omega_{\nu}, H, W \in \mathfrak{h}$ one has $\left\langle J,\left[h_{\nu, H}(\lambda), h_{\nu, W}(\lambda)\right]\right\rangle=0$. Inserting into it the asymptotic expressions from Proposition 1 and equating to zero the coefficients in front of $\lambda^{-N}$ we get

$$
\sum_{k=0}^{N}\left\langle J,\left[\Lambda_{-}^{N-k} q_{H}, \Lambda_{-}^{k} q_{W}\right]\right\rangle=0 .
$$

In the left hand side of this expression we can use the identities of the type (49) for all the terms except the first and the last one in order the obtain terms in which $\Lambda_{-}$ acts only on $q_{H}$. Thus we obtain

Lemma 6. For $N \geq 0, H, W \in \mathfrak{h}$

$$
N\left\langle J,\left[q_{H}, \Lambda_{-}^{N} q_{W}\right]\right\rangle+\left\langle J,\left[\Lambda_{-}^{N} q_{H}, q_{W}\right]\right\rangle=\mathrm{i} \partial_{x} \mathfrak{F}_{2}(H, W ; N-1)
$$

where $\mathfrak{F}_{2}(H, W ; N-1)$ is a polynomial with constant coefficients depending on $\left\langle\mathrm{I}_{-} \Lambda_{-}^{k} q_{H}, \mathrm{I}_{-} \Lambda_{-}^{s} q_{W}\right\rangle$ and $\left\langle\Lambda_{-}^{k} q_{H}, \Lambda_{-}^{s} q_{W}\right\rangle, 0 \leq s, k \leq N-1$.

Finally, combining the two lemmas we get

Proposition 7. For $N \geq 0, H, W \in \mathfrak{h}$

$$
\left\langle J,\left[q_{H}, \Lambda_{-}^{N} q_{W}\right]\right\rangle=\mathrm{i} \partial_{x} \mathfrak{F}_{3}(H, W ; N-1)
$$

where $\mathfrak{F}_{3}(H, W ; N-1)$ is a polynomial wiith constant coefficients on $\left\langle\mathrm{I}_{-} \Lambda_{-}^{k} q_{H}, \mathrm{I}_{-} \Lambda_{-}^{s} q_{W}\right\rangle$ and $\left\langle\Lambda_{-}^{k} q_{H}, \Lambda_{-}^{s} q_{W}\right\rangle, 0 \leq s, k \leq N-1$.

Now we have

Theorem 8. For arbitrary $H, W \in \mathfrak{h}$ and arbitrary natural $N$ the expressions $\left\langle J,\left[q_{H}, \Lambda_{ \pm}^{N} q_{W}\right]\right\rangle$ are $x$-derivatives of local functions on $q, q_{x}, \ldots$

Proof: We shall prove the theorem for $\left\langle J,\left[q_{H}, \Lambda_{-}^{N} q_{W}\right]\right\rangle$, as we have seen then it will follow for $\left\langle J,\left[q_{H}, \Lambda_{+}^{N} q_{W}\right]\right\rangle$. We shall use induction.

I. $N=1$. We have that

$$
\Lambda_{-} q_{H}=\operatorname{iad}_{J}^{-1} \partial_{x} q_{H}+\operatorname{ad}_{J}^{-1} \pi_{0}\left[q, q_{H}\right]+\operatorname{iad}_{J}^{-1} \operatorname{ad}_{q} \mathrm{I}_{-} q_{H} .
$$


It can be verified that $\left(\mathrm{id}-\pi_{0}\right) \operatorname{ad}_{q} \operatorname{ad}_{J}^{-1} \operatorname{ad}_{q}\left(\mathrm{id}-\pi_{0}\right)=0$. As a consequence, $\Lambda_{-} q_{H}=\operatorname{iad}_{J}^{-1} \partial_{x} q_{H}+\operatorname{ad}_{J}^{-1} \pi_{0}\left[q, q_{H}\right]$ and

$$
\left\langle J,\left[q_{W}, \Lambda_{-} q_{H}\right]\right\rangle=\left\langle[W, q], \operatorname{iad}_{J}^{-1} \partial_{x} q_{H}+\operatorname{ad}_{J}^{-1} \pi_{0}\left[q, q_{H}\right]\right\rangle .
$$

But due to the identity $\left(\mathrm{id}-\pi_{0}\right) \operatorname{ad}_{q} \operatorname{ad}_{J}^{-1} \operatorname{ad}_{q} \operatorname{ad}_{J}^{-1} \operatorname{ad}_{q}\left(\mathrm{id}-\pi_{0}\right)=0$ we have

$$
\begin{aligned}
\left\langle[W, q], \operatorname{ad}_{J}^{-1} \pi_{0}\left[q, q_{H}\right]\right\rangle & =-\left\langle\operatorname{ad}_{J}^{-1}[W, q], \pi_{0}\left[q, q_{H}\right]\right\rangle=\left\langle q_{W},\left[q, q_{H}\right]\right\rangle \\
& =-\left\langle W, \operatorname{ad}_{q} \operatorname{ad}_{J}^{-1} a d_{q} \operatorname{ad}_{J}^{-1} \operatorname{ad}_{q} H\right\rangle=0
\end{aligned}
$$

and finally we obtain

$$
\left\langle J,\left[q_{W}, \Lambda_{-} q_{H}\right]\right\rangle=\left\langle[W, q], \operatorname{iad}_{J}^{-1} \partial_{x} q_{H}\right\rangle=-\frac{\mathrm{i}}{2} \partial_{x}\left\langle q_{W}, q_{H}\right\rangle
$$

Thus for $N=1$ the statement is true.

II. The inductive step $N-1 \rightarrow N$ is easily obtained using Proposition 7. The Theorem is proved.

Corollary 9. For every $H \in \mathfrak{h}$ and $N=0,1,2, \ldots$ the expressions $\Lambda_{ \pm}^{N} q_{H}$ are local function on $q, q_{x}, \ldots$ and thus all the equations: $\operatorname{ad}_{J}^{-1} \partial_{t} q=\Lambda_{ \pm}^{N} q_{H}$ are local.

Proposition 10. The conservation laws $d_{H, s}, H \in \mathfrak{h}$, see (43), have local densities.

Finally, as it can be readily verified, arguments of the type we have used already show that the expressions of the type $\left\langle J,\left[\Lambda_{-}^{k} q_{H}, \Lambda_{-}^{s} q_{W}\right]\right\rangle$ are $x$-derivatives of local functions. Then we have another important result

Proposition 11. The conservation laws $d_{W, s}$ are in involution with respect to a hierarchy of symplectic forms.

Indeed, as it is well-known the NLEEs related to $L$ are Hamiltonian with respect to the following hierarchy of symplectic forms, see [9]

$$
\omega_{m}(X, Y)=\int_{-\infty}^{+\infty}\left\langle X, \Lambda_{ \pm}^{m} \operatorname{ad}_{J}^{-1}(Y)\right\rangle \mathrm{d} x, \quad m=0,1,2 \ldots
$$

Taking into account the form of $\omega_{m}$ the result easily follows. Our final remark is that in all the proof of this subsection we used some identities that we derived from the asymptotic properties of the functions $h_{\nu, H}$ and thus they do not depend on the spectrum of the linear problem for concrete $q$ and are not limited say to the case of small potential. 


\section{Acknowledgements}

The author is grateful to NRF South Africa Incentive Grant 2013 for the financial support.

\section{References}

[1] Ablowitz M., Kaup D., Newell A. and Segur H., The Inverse Scattering Method - Fourier Analysis for Nonlinear Problems, Studies in Appl. Math. 53 (1974) 249-315.

[2] Beals R. and Coifman R., Scattering and Inverse Scattering for First Order Systems, Comm. Pure \& Apppl. Math. 37 (1984) 39-90.

[3] Beals R. and Coifman R., Inverse Scattering and Evolution Equations, Commun. Pure \& Appl. Math. 38 (1985) 29-42.

[4] Beals R. and Coifman R., Scattering and Inverse Scattering for First Order Systems II, Inverse Problems 3 (1987) 577-593.

[5] Caudrey P., The Inverse Problem for a General $N \times N$ Spectral Equation, Physica D 6 (1982) 51-56.

[6] Drinfeld V. and Sokolov V., Lie Algebras and Korteweg-de Vries type Equations, (in Russian) VINITI, Contemporary Problems of Mathematics. Recent Developments 24, Moscow 1984, pp 81-180.

[7] Faddeev L. and Takhtadjan L., Hamiltonian Method in the Theory of Solitons, Springer, Berlin 1987.

[8] Gerdjikov V., Generalized Fourier Transforms for the Soliton Equations. Gauge-covariant Formulation, Inverse Problems 2 (1986) 51-74.

[9] Gerdjikov V., Vilasi G. and Yanovski A., Integrable Hamiltonian Hierarchies - Spectral and Geometric Methods, Springer, Heidelberg 2008.

[10] Gerdjikov V. and Yanovski A., Generating Operator and Locality of the Conservation Laws. The Case of Zakharov-Shabat Linear Problem (In Russian), JINR Communication R5-82-242, Dubna (1985).

[11] Gerdjikov V. and Yanovski A., Completeness of the Eigenfunctions for the Caudrey-Beals-Coifman System, J. Math. Phys. 35 (1994) 3687-3721.

[12] Gerdjikov V. and Yanovski A., On Soliton Equations with $\mathbb{Z}_{h}$ and $\mathbb{D}_{h}$ Reductions: Conservation Laws and Generating Operators, J. Geom. Symmetry. Phys. 31 (2013) 57-92.

[13] Goto M. and Grosshans F., Semisimple Lie Algebras, M. Dekker, New York 1978. 
[14] Gürses M., Karasu A. and Sokolov V., On Construction of Recursion Operators from Lax Representation, J. Math. Phys. 40 (1999) 6473-6490.

[15] Mikhailov A., The Reduction Problem and Inverse Scattering Method, Physica 2D N1/2 (1981) 73-117.

[16] Shabat A., The Inverse Scattering Problem for a System of Differential Equations (In Russian), Functional Annal. \& Appl. 9 (1975) 75-78;

Shabat A., The Inverse Scattering Problem (In Russian), Diff. Equations 15 (1979) 1824-1834.

[17] Yanovski A., Recursion Operators and Expansions Over Adjoint Solutions for the Caudrey-Beals-Coifman System with $\mathbb{Z}_{p}$ Reductions of Mikhailov Type, J. Geom. Symmetry Phys. 30 (2013) 105-120.

[18] Yanovski A., Geometry of the Recursion Operators for Caudrey-BealsCoifman System in the Presence of Mikhailov $\mathbb{Z}_{p}$ Reductions, J. Geom. Symmetry Phys. 25 (2012) 77-97.

[19] Zakharov V. and Shabat A., A Scheme for Integrating Nonlinear Evolution Equations of Mathematical Physics by the Inverse Scattering Method I, Funkts. Anal. Prilozhen. 8 (1974) 43-53.

[20] Zakharov V., Manakov S., Novikov S. and Pitaevskii L., Theory of Solitons: The Inverse Scattering Method, Plenum, New York 1984.

Alexandar Yanovski

Department of Mathematics

and Applied Mathematics

University of Cape Town

7700 Rondebosch, Cape Town

SOUTH AFRICA

E-mail address: Alexandar. Ianovsky@uct.ac.za 